\title{
An Air-to-Liquid MEMS Particle Sampler
}

\author{
Amish Desai, Sang-Wook Lee, Yu-Chong Tai \\ California Institute of Technology, MS 136-93, \\ Pasadena, California, 91125 USA
}

\begin{abstract}
In order to have a working bio-particle analysis system, a method of capturing the particles from the air into the liquid is required. Here, we report a complete MEMS system that includes an air-to-liquid MEMS interface (made of glass and PDMS) for airborne bioparticle $(<10 \mu \mathrm{m})$ analysis, and demonstrate its successful integration with our DEP(dielectrophoretic) particle transportation[1] and active filter membrane[2] technology. Two types of air-to-liquid interfaces were investigated. The first, consisted of a stationary meniscus with moving particles; and second, stationary particles with an oscillating liquid meniscus. Due to large interfacial forces required in penetrating the liquid meniscus, the first design performed inadequately. However, these roadblocks were eliminated in the second technique and demonstrated as a working system.
\end{abstract}

\section{INTRODUCTION}

There is a growing need for a hand-held, automated environmental monitoring system for the detection of harmful bio-aerosols such as toxins, viruses, and spores. Successful detection of these types of particles requires three steps - collection, transport, and analysis. For airborne bio-particles, analysis is problematic because many of the sensitive and standardized bio-analytical techniques (such as florescence, PCR, ESI-MS etc) only work in the liquid medium. Consequently, engineering of an air-liquid interface is crucial for transport of the collected particles from the air into the liquid medium. In the past, the transport of airborne particles into a liquid medium has primarily been accomplished by manual pipetting and washing [3], 30cm long denuders [4], and cyclone chambers. However, these types of traditional techniques become impractical for such a MEMS hand-held system that requires microliter volumes, miniature components, and mechanical robustness. To our knowledge, no MEMS solution to this airborne sample collection problem has been published before.

\section{THE AIR LIQUID INTERFACE}

In this work, a solution to this interface problem was engineered and integrated with the MEMS particle transportation technology developed in our prior work[1]. The first generation design consisted of a glass chip with hydrophobic channels bonded to the particle transport chip. The focus, here, was to design the interface channel small enough so that the surface tension forces would prohibit liquid leakage outward, but allow particles to be transported across the airliquid interface. After discovering problems with this first scheme (stationary meniscus and moving particles), the interface problem was tackled with a different approach. In this second approach, instead of moving particles and a stationary interface, we demonstrate a working particle-to-liquid collection mechanism with a moving meniscus and stationary particles. Borosilicate glass particles $(8 \mu \mathrm{m})$ were used for all experiments since they are typical test carriers for surface deposited biological agents. With these particles, the feasibility of this technique was established together with our micromachined active membrane filter published in [5]. Although particle transport efficiency decreased greatly on the perforated filter membrane, the feasibility for an airborne bioparticle sampling system was demonstrated. Some of the issues explored here were the following: 1) Stability of the air-liquid interface, 2) Control of the fluid meniscus 3) Performance of the transportation scheme in liquid, and 4) Integration of particle transportation technology with a fluidic interface chip and an active micromachined membrane filter (with embedded electrodes).

\section{STATIONARY MENISCUS INTERFACE}

The schematic for the particle interface design is shown in Fig. 1. The glass chip (Fig. 2) contains etched channels, which simulate a liquid system in which the particles could be distributed and analyzed. The three " $T$ " channels (shown as dashed lines) contain the liquid meniscus interfaces which are connected to a larger central flow channel. Using the DEP force, the particles would be transported from the electrode across the interface into the bulk liquid.

DEP control of particles in liquid has been demonstrated by [6][7][8] who have shown that in contrast to the high voltage, low frequencies required in air, low voltages and high frequencies are required for particle transport in liquid. In fact, DEP particle motion in liquid has been shown to be much more robust and efficient than in air. Table 1 illustrates the different regimes demonstrated by various groups. In our case, two circuits were connected to the electrodes so that when the particles entered the liquid, the proper 
Table 1: DEP electrical requirements for different media.

\begin{tabular}{|l|l|l|l|}
\hline Reference & Voltage & Frequency & Medium \\
\hline Desai et al. [1]. & $100-200 \mathrm{~V}$ & $5-100 \mathrm{~Hz}$ & Air \\
\hline Fuhr et al. $[6]$ & $1-5 \mathrm{~V}_{\mathrm{pp}}$ & $1 \mathrm{Mhz}$ & Conductive liquid (10mS/m) \\
\hline Lee et al. $[7]$ & $120-140 \mathrm{~V}$ & $1-1000 \mathrm{~Hz}$ & Corn oil $\left(10^{-11} \mathrm{~S} / \mathrm{m}\right)$ \\
\hline Morgan et al. $[8]$ & $10 \mathrm{~V}_{\mathrm{pp}}$ & $500 \mathrm{kHz}$ & Conductive liquid $(150 \mathrm{mS} / \mathrm{m})$ \\
\hline
\end{tabular}

waveform (circuit) would be activated for either air or water.

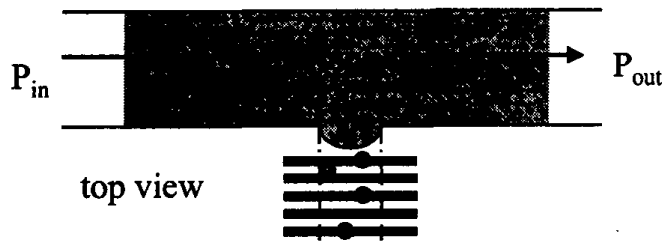

Figure 1: $1^{\text {st }}$ Particle air to liquid collection schematic

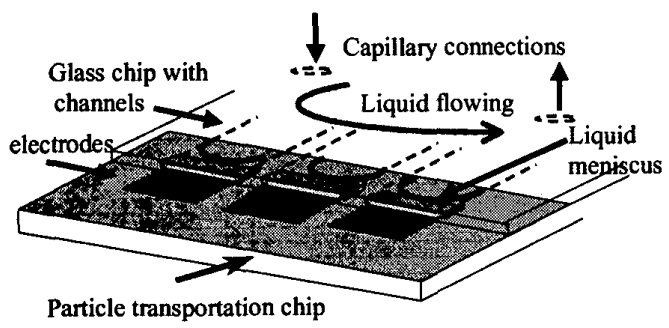

Figure 2: $1^{\text {st }}$ Particle air to liquid collection scheme

To work, this scheme requires that the midstream pressure, $P_{m}$, of the flow at the interface be less than the capillary pressure, $P_{c}$. The governing aspects of this design are the Young-Laplace and the HagenPoiseuille equations [9]. From the Young-Laplace equation, we are able to predict the maximum pressure that can be present near the opening. Similarly, the Poiseuille equation helps us predict the pressure drop in the central channel for a given flow rate. The Young-Laplace equation, Eq. 1, is a function of the surface tension of the fluid, $\sigma$, the capillary radius, $r$, and the meniscus contact angle, $\theta$. Assuming the meniscus is a section of a spherical surface that intersects the capillary at a finite contact angle, we have the static case [10],

$$
P=(2 \sigma / r) \cos (\theta) \text {. }
$$

Though Eq. 1 is valid only for circular openings, it is sufficient to demonstrate the features of this design. The elaborate closed form solution for our case, a rectangular slit has been derived by Kagen and Pinczewski [11] in a lengthy derivation. They show that closed form solution for the pressure, $\mathrm{P}$, for the rectangular slit falls below that for the circular channels. Consequently, the Young-Laplace equation represents the "best case" solution. To better understand the maximum possible interface pressures, Eq. 1 has been plotted in Fig. 2 for pure water and a highly hydrophobic capillary wall $\left(\theta \sim 180^{\circ}\right)$.

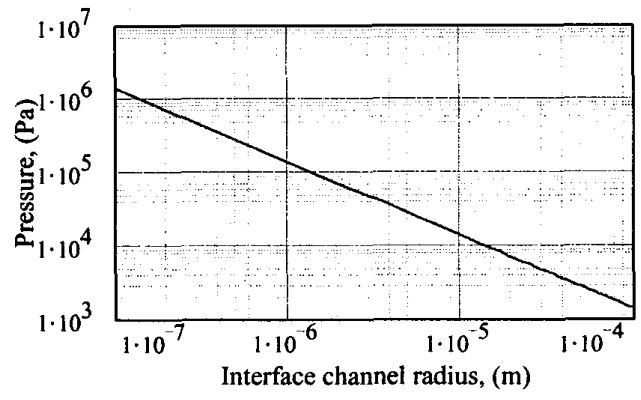

Figure 3: Maximum meniscus pressures for water vs. interface channel radius.

The Poiseuille flow equation relates flow rate, $Q$, to the pressure drop, $\Delta \mathrm{P}$, the pipe radius, $r$, viscosity, $\mu$, and the distance from the interface to the exit, $\mathrm{L}$.

$$
\mathrm{Q}=\Delta \mathrm{P} \pi \mathrm{r}^{4} /(8 \mu \mathrm{L})
$$

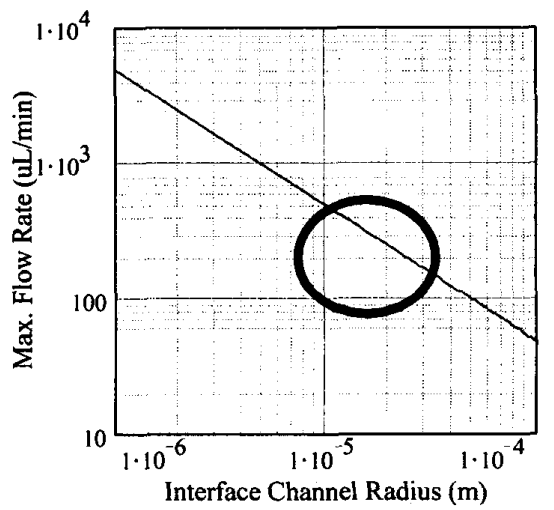

Figure 4: Idealized flow rates possible for given interface channel sizes

Substituting Eq. 1 for $\Delta \mathrm{P}$ in Eq. 2 and making the following assumptions, we can estimate the best case flow rates possible for given interface channel sizes. (Fig. 4) The following values, $5 \mathrm{~mm}$ and $50 \mu \mathrm{m}$, were 
chosen for $\mathrm{L}$ and $\mathrm{r}$, respectively. Note that Fig. 4 represents an ideal case, where the contact angle is $180^{\circ}$, the interface channel surface is perfectly smooth and clean, and the interface channel is circular. In our case, the channels are rectangular slits. Furthermore, the exit pressure due to external piping and constrictions lower the maximum possible flow rates even further. Therefore, we expect the order of the allowable flow rates to be correct, but certainly two to three times lower than these idealized flow rate regime shown in Fig. 4.
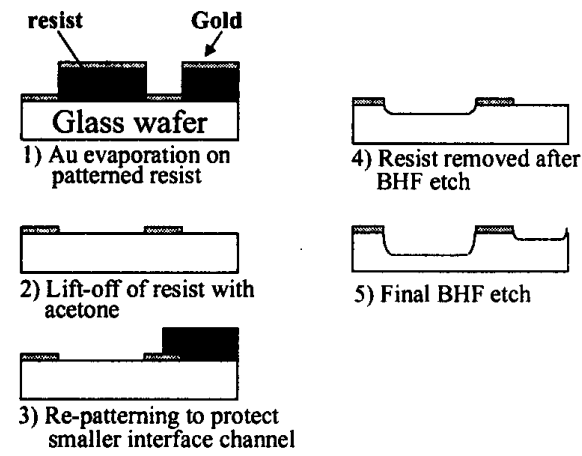

Figure 5: Glass interface channel fabrication steps.

\section{STATIONARY INTERFACE FABRICATION}

Fabrication of the interface chip required two glass etching steps to create the deeper central flow channel and the shallower interface " $T$ " channels. Evaporated gold acted as the masking layer and buffered hydroflouric acid (BHF), as the etchant. Photographs of various designs are shown in Fig. 6. The designs have curved, tapered, and widened channel entrances to study which types could sustain the fluid meniscus pressures and which types allowed more particles to be captured. This lift-off process is illustrated in Fig. 5. The etch rate of the glass in BHF was roughly $1 \mu \mathrm{m} / \mathrm{min}$. Inlet and outlet holes were drilled mechanically with a $700 \mu \mathrm{m}$ diameter drill bit.

To make the interface channels hydrophobic, a Teflonlike polymer film was plasma deposited as the final step. In the past, fluorine-based gases have been utilized in plasma processing for their ability to selectively etch silicon. But, under certain conditions these gases can be polymerized to form thin films on a substrate. For the interface chip, the fluorocarbon gas, $\mathrm{CHF}_{3}$, was polymerized in the plasma at the following conditions: $170 \mathrm{~W} 300 \mathrm{~m}$ Torr, $2.7 \mu \mathrm{m} / \mathrm{hr}$.

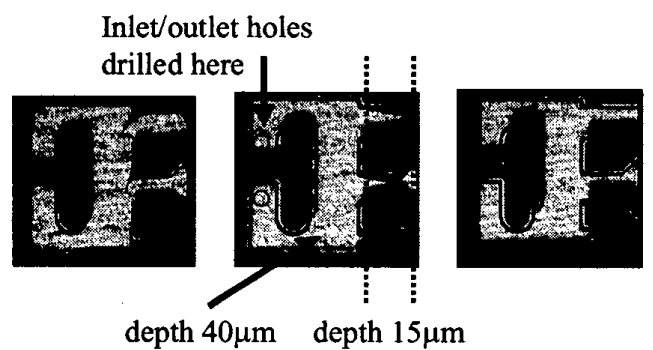

Figure 7: Various glass interface channel designs

In addition to the $\mathrm{CHF}_{3}$ film, hexamethyldisilizane (HMDS) vapor treatment was also used to make the glass surface hydrophobic. Selective deposition of the hydrophobic films only in the interface channel area was done with a physical mask because any type of photoresist processing such as developer and acetone rinsing destroyed the hydrophobicity of the polymer film surface (AZ4400 and AZ developer).

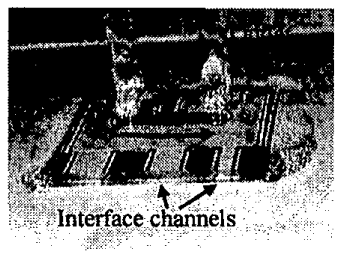

Figure $8: 1 \mathrm{~cm} \mathrm{x} 1 \mathrm{~cm}$ glass interface die bonded to a glass substrate for initial testing

After coating the channel surfaces, the interface chip was bonded to a glass slide for the initial experiments (Fig. 6). The inlet pipe was connected to a digitally controlled syringe pump to simulate a micromachined liquid pumping system. The interface chips were bonded to a particle transportation chip (Fig. 7), after suitable control of the meniscus was achieved. The electrode chips were redesigned to include large $5 \times 3$ $\mathrm{mm}$ electrode arrays on which adjacent dies on either side were left blank to leave room for bonding the glass channel chip.

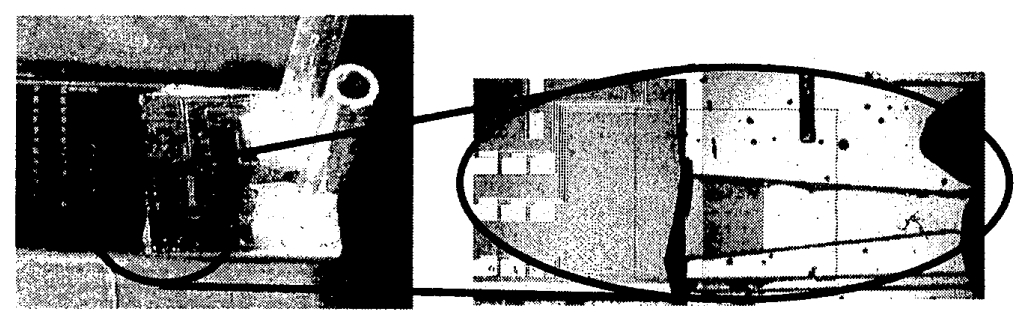

Figure 6: $1 \mathrm{~cm} \mathrm{x} 1 \mathrm{~cm}$ glass interface die bonded to a glass substrate for initial testing 


\section{STATIONARY INTERFACE RESULTS}

The first experiments with just the interface die bonded to glass proved to be difficult. The epoxy bonding step was problematic and decreased yield. Experiments were conducted by slowly increasing the flow rate until the meniscus broke down for various coatings (bare glass, HMDS, and the $\mathrm{CHF}_{3}$ fluoro-polymer). As expected, uncoated interface channels only withstood flow rates on the order of $0.1-1 \mu \mathrm{L} / \mathrm{min}$. The more hydrophobic the interface, the higher the flow rate achieved. For example, the silane coated dies attained flow rates on the order of $1-5 \mu \mathrm{L} / \mathrm{min}$, and the $\mathrm{CHF}_{3}$ Teflon dies could withstand flow rates of 50 $100 \mu \mathrm{L} / \mathrm{min}$ which was in good agreement with the flow regime in Fig. 4. Unfortunately, the fluid meniscus was only stable for $\sim 30 \mathrm{~min}$. Once the surface had been contaminated, the level of hydrophobicity decreased, and the chip would fail. Furthermore, the position of the meniscus was difficult to control due to the small dead volume of the interface channel $(0.045 \mu \mathrm{L})$. Nevertheless, the next step was to experiment with working electrodes and moving silica glass particles.

Experiments with the combined electrode and interface chips proved to be failures for two reasons. Firstly, particles transported in air on the electrodes could not travel past the liquid meniscus, and conglomerated at the boundary. Secondly, the high voltage $(150 \mathrm{~V}$, $50 \mathrm{~Hz}$ ) required for particle transportation in air caused electrolysis of the water present on the electrodes even though the electrodes were well insulated by LTO and Teflon. The bubbles in the water would form within 10 seconds and destroy the electrodes. This result made it clear that during the high voltage, low frequency transportation, no water could be present on the electrodes. The voltage required turned out to be an order of magnitude higher than the voltages demonstrated in prior work in this field (Table 1). $50 \mathrm{~V}$ at $500 \mathrm{Mhz}$ were required to move the silica particles in water in our system.
Although this meniscus based interface design failed to work, valuable information on properties of surfaces, stability of air-liquid interface, and the performance envelope of the DEP force was acquired. The $\mathrm{CHF}_{3}$ Teflon-like film was the most hydrophobic, and as expected, the highest flow rate $(100 \mu \mathrm{L} / \mathrm{min})$ was achieved for the $15 \mu \mathrm{m} \times 1 \mathrm{~mm}$ which was three times slower than for the idealized case (see Fig. 3). Because of the rectangular channel, and unaccounted exact pressure drops in the outlet side of the chip, we expected this slower flow rate. On the other hand, the DEP scheme in both environments - air and water, on the same set of electrodes proved unfeasible. From the observations, it was clear that the water could not be exposed to the high voltage, low frequency scheme needed for particle transport in air. Moreover, even if the electrolysis problem could be solved, the DEP force was insufficient to drive the particles through the fluid meniscus. A different approach was needed.

\section{MOVING INTERFACE DESIGN}

Because of these two obstacles, a different method was taken to transport the particles across the air-liquid interface. Since the DEP force was insufficient to provide the penetration force, the energy of a moving meniscus was used capture the particles instead. The second generation air-to-liquid capture scheme is shown in Fig. 9. The DEP driving circuit $(160 \mathrm{~V}, 10-$ $50 \mathrm{~Hz}$ ) is turned on until particles are transported inside the interface channel (Step 1). Next, the power is turned off, and then controlled oscillations of the meniscus capture the particles (Step 2). In step three, the meniscus is sucked back so that no liquid is present on the electrodes, and the electrode circuit is turned back on. This cycle is repeated.

For demonstration of the concept, the pumping and sucking was done manually. Though in the future, one could envision integration with micro-pumps and valves. Furthermore, an active filter was designed onto a section of the particle transportation chip. The active filter was a modification of the electrode fabrication process described previously but with

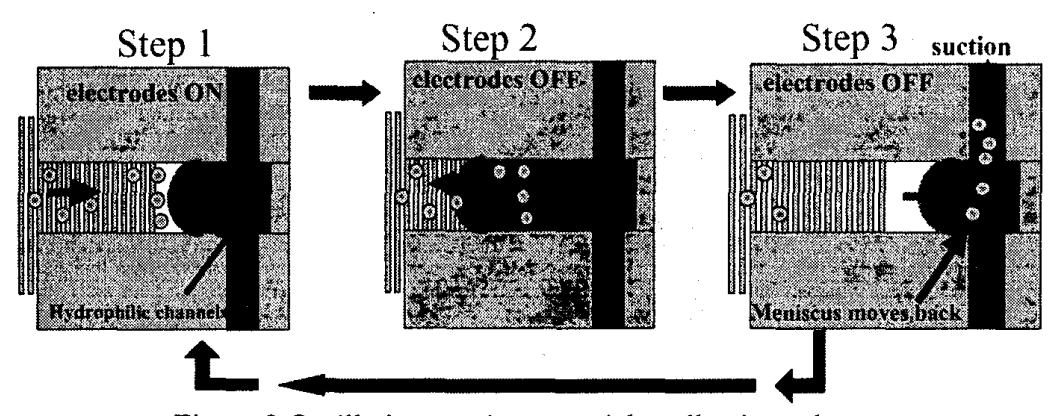

Figure 9:Oscillating meniscus particle collection scheme 


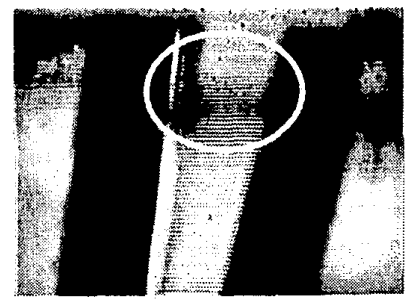

1. Particles moved into interface area

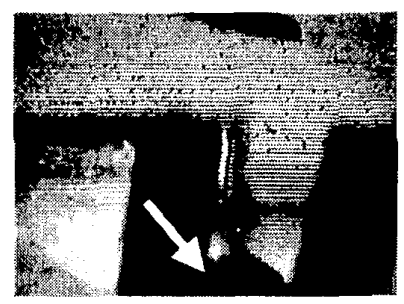

2. Meniscus wash step

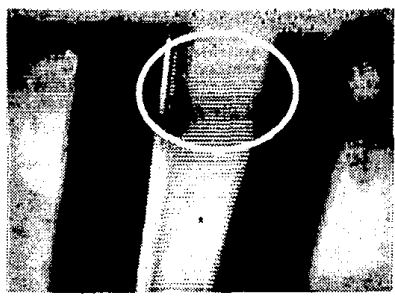

3. Interface area cleared

Figure 11: Video snapshots of particle capture sequence.

backside wafer processing to realize the electrodes on a perforated silicon/silicon nitride filter membrane. Fig. 10 shows a new transportation chip layout $(1 \mathrm{~cm} \mathrm{x}$ $1 \mathrm{~cm})$ redesigned with large array of various electrodes and filter areas with their respective sizes. The first number in the label ("R" or "P") refers to the electrode width, and the second number refers to the space in between the electrodes. To increase collection efficiency (with respect to the linear arrays), the radial electrodes, depicted here, serve as particle concentrators - grouping the particles in one central area.

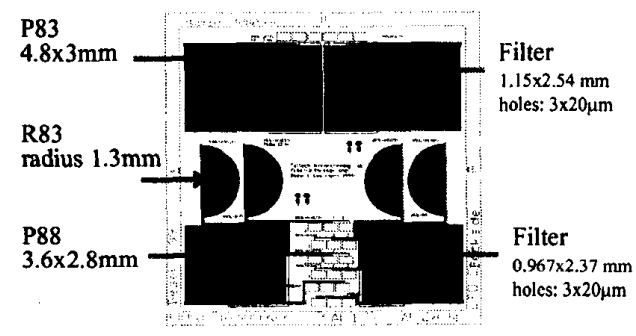

Figure 10: Integrated filter and particle transport layout $(1 \mathrm{~cm} \times 1 \mathrm{~cm})$

\section{MOVING INTERFACE FABRICATION}

A silicone rubber (Sylgard 184 ${ }^{\circ}$ ) channel is clamped to the filter-electrode chip.(Fig. 12,13) The fabrication of the electrode-filter chip which is a combination of $\mathrm{KOH}$ etching, deep reactive ion etching, and CHF3 plasma deposited polymer coatings is detailed in our prior work [2]. Using the silicone channel eliminates the difficult bonding to the plasma Teflon coated electrode chip as well as ensuring sealing over a nonplanar surface. Combining the filter-plus-electrode structure complicated bonding of the interface channels with the transportation chip because the spinon Teflon AF1601S could not be used as the optimal dielectric layer. Because of the filter membrane, no spin on type processing could be done on the wafer. Instead, a conformal $\mathrm{CHF}_{3}$ polymer was plasma deposited as the insulation.

\section{Top View}

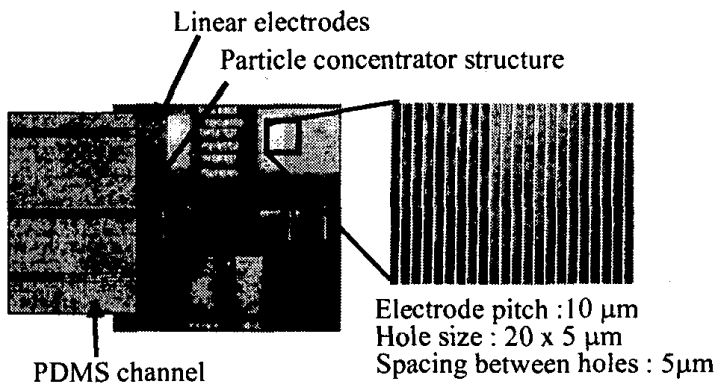

Figure 12: Integrated filter and particle transport layout $(1 \mathrm{~cm} \times 1 \mathrm{~cm})$

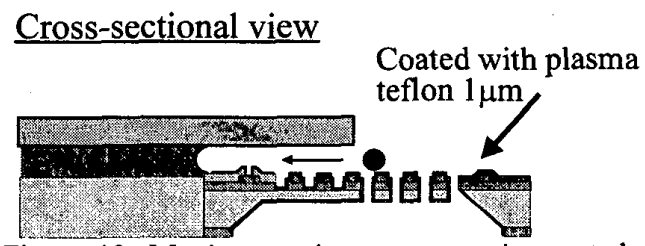

Figure 13: Moving meniscus concept integrated with the active filter membrane

Details of this active filter fabrication can be found in work by Lee et al. [1] In any case, bonding to Teflonlike materials is generally troublesome; so the interface channel was molded with a flexible, transparent silicone elastomer, Sylgard ${ }^{\otimes} 184$ (Dow Corning Corp.), and clamped down to the particle transportation chip. A schematic of the cross section and side view of the assembled device is illustrated in Fig. 13. A video snapshot of a working prototype is shown in Fig. 14. Much larger than the $\sim 50 \mu \mathrm{m}$ glass channels, the $500 \mu \mathrm{m}$ diameter elastomer channel allowed better control of the meniscus position.

\section{MOVING INTERFACE RESULTS}

For the moving meniscus collection technique, experiments were successfully performed for the linear electrodes as well as for the radial structures $(8 \mu \mathrm{m}$ silica glass particles tested). A series of video snapshots of one experiment is depicted in Fig. 10. The velocity of the oscillating meniscus ranged from 
$1-3 \mathrm{~mm} / \mathrm{s}$.

The collection efficiency was only about 5-10\% because of three factors. First, there was a significant drop in efficiency due to the $\mathrm{CHF}_{3}$ polymer coating instead of the spin-on Teflon. Secondly, the filter holes and structure also reduced efficiency from the normally observed $80-90 \%$ levels on the standard electrode grid. Finally, the particle contact with the liquid meniscus was limited by the interface opening width of $500 \mu \mathrm{m}$. The rest of the particles collected on the edges of the silicone. Regarding this problem, the particle radial structures worked much better to concentrate all the particles at one point. (Fig. 14)

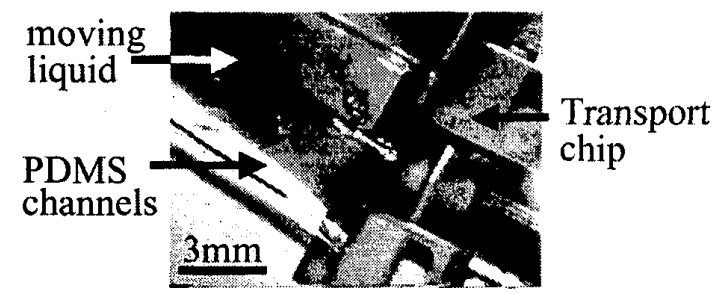

Figure 14: Video image of interface chip on radial transportation structure.

One problem during testing was the long-term hydrophobicity of the plasma Teflon surface. After exposure to water, the Teflon surface started to retain micro-droplets of water $(<2 \mu \mathrm{m})$. This liquid residue affected the shape of the meniscus, and also reduced particle transportation efficiency in that area. In other words, particles would begin to get stuck just outside the travel of the meniscus. In addition, the whole experiment failed, if the liquid accidentally was pushed out beyond the interface channel onto the rest of the electrode grid.

\section{CONCLUSION}

Two different particle air-to-liquid collection schemes were explored. The first type involved two regimes of voltages and frequencies but failed because the strength of the DEP force was too small to penetrate the liquid meniscus, and because of electrolysis of water on the electrodes. Successful collection was demonstrated through an entirely different type of collection technique- in which the liquid meniscus was moving and not the particles. With this method, the problems of the first type were circumvented. In addition, a membrane filter structure was integrated with the transportation technology and a new radial design demonstrated improved collection efficiency. To summarize, the feasibility of a MEMS airborne particle sampling system has been established. This novel micromachined air-liquid collection system is only a first solution for this complex particle collection and interface problem, but it paves the way for the creation of a truly automated hand-held airborne bio- particle analysis system.

\section{ACKNOWLEDGMENTS}

The funding for this project was provided by DARPA, MicroFlumes program under contract No. N66001-96C-83632. The authors would like to thank Trevor Roper and Ellis Meng for their help.

\section{REFERENCES}

[1] A. Desai, S. W. Lee, and Y.C.Tai. "A MEMS Electrostatic Particle Transportation System." The Eleventh Annual International Workshop on Micro Electro Mechanical Systems (MEMS '98), Jan 1998.

[2] S.W. Lee, J.M. Yang, Y.C. Tai, and C.M. Ho, "Electrostatically Active Microfilters For Automated Airborne Particle Collection," Proceedings of Transducers '99, IEEE1999.

[3] C.S. Cox, C.M. Wathes, Bioaerosols Handbook, Lewis Publishers, Boca Raton, 1995.

[4] P.K. Dasgupta, L. Ni, S.K. Poruthoor, D.C. Hindes, “A Multiple Parallel Plate Wetted Screen Diffusion Denuder for High-Flow Air Sampling Applications," Analytical Chemistry, Vol. 69, 5018-5023.

[5] S.W. Lee, J.M. Yang, Y.C. Tai, and C.M. Ho, "Electrostatically Active Microfilters For Automated Airborne Particle Collection," Proceedings of Transducers '99, IEEE1999.

[6] G. Fuhr, T. Muller, Th. Schnelle, R. Hagedorn, A. Voigt, and S. Fiedler, "Radio Frequency Microtools for Particle and Live Cell Manipulation," Natur Wissenschaften Aufsatze, Springer-Verlag, 1994.

[7] S.W. Lee, Y-W Kim, and Y-K Kim, "Determination of Dielectric Constant of Dielectric Particles Using Negative Dielectrophoresis," Conference Proceedings on Electrical Insulation and Dielectric Phenomena," San Francisco, October 20-23, 1996.

[8] H. Morgan, N.G. Green, M. P. Hughes, and W. Monaghan, T.C. Tan, "Large-area travelling-wave dielectrophoresis particle separator," Journal of Mechanics and Microengineering, Vol. 7, Pg. 6570, 1997.

[9] F.M. White, Viscous Fluid Flow, McGraw Hill, New York, 1991.

[10] Middleman, S., "Modeling Axisymmetric Flows: Dynamics of Films, Jets, and Drops," Academic Press, San Diego, 1995.

[11] M. Kagan and W.V. Pinczewski, "NOTE Meniscus in a Narrow Slit," Journal of Colloid and Interface Science, Vol. 180, Pg. 293-295, 1996. 